\title{
A Survey of Detection of Cognitive Impairment using Non-invasive Indicators
}

\author{
Shridevi Karande \\ PhD Scholar, School of Computer Science and \\ Engineering \\ Dr.Vishwanath Karad MIT World Peace University \\ Pune, India
}

\author{
Vrushali Kulkarni, PhD \\ Professor, School of Computer Science and \\ Engineering \\ Dr.Vishwanath Karad MIT World Peace University \\ Pune, India
}

\begin{abstract}
Cognitive Impairment is a stage where a person faces difficulty in processing information, remembering, learning new things, concentrating or making decisions affecting their day to day life. These impairments range from mild to severe stage and in the long term they lead to Dementia and Alzheimer's disease. A person may have natural decline of cognition over the age and the nature of impairment changes from person to person. It's important to detect and measure these changes from time to time. The heterogeneous nature of Cognitive impairment and the natural decline of cognition over the age makes its detection more difficult. The review presented here is a study of various non-invasive methods such as neuropsychological tests, speech and eye dynamics used to measure cognitive and behavioural changes. These methods are used in preclinical frontline screening and diagnosis of impairment and they have their have their own relative accuracy when used separately. This review explores a multi-modal approach of combining these cognitive and behavioural markers to improve detection accuracy.
\end{abstract}

\section{General Terms}

Cognitive Impairment, Cognitive Decline.

\section{Keywords}

Dementia, Alzheimer's disease(AD), Neuropsychological Assessment, Cognitive domain.

\section{INTRODUCTION}

Cognitive impairment is a decline of Cognitive function such as executive function, gnosis, language, orientation, attention, praxis, memory, visual-spatial perception and social cognition. Cognitive level progressively declines through several stages as follows:

I) Preclinical Stage is where no symptoms are noticed in a person but brain changes have begun.

II) Mild cognitive Impairment (MCI) is stage where cognitive decline is greater than expected for an individual's age and education level but that does not interfere notably with activities of daily life. MCI is often a challenging phase and remains undiagnosed.

III) Dementia: Prolonged cognitive impairments lead to dementia which is a significant decline in areas such as cognitive skills (memory, speech, thinking), functional abilities (daily activities such as dressing, eating, walking), mood and behaviour. Alzheimer's disease is one common form of Dementia. AD is a progressive, degenerative disorder that attacks brain's nerve cells, or neurons, resulting in loss of memory, thinking and language skills, and behavioral changes.
As per World Health Organization report, the number of people living with dementia worldwide is currently estimated as 47 million. It is projected to increase to 75 million by 2030 and number of cases of dementia are estimated to almost triple by 2050 . In India, the current older population of 60 million is projected to exceed 227 million by 2050 , an increase of nearly 3.7 times from today [1]. 4.1 million Of these dementia cases are found in India. This is approximately $10 \%$ of global dementia cases. Nearly half of global dementia people will live in Asia by 2050[2].

\subsection{Importance of Detection}

MCI stage is the earliest stage where the patient or the family members observe decline in cognitive functionality. In recent years, even younger people have been found to have developed dementia which is about $2-8 \%$ of the total dementia cases. Dementia often is seen as a socially undesired trait, leading to discrimination and social isolation [2]. Due to fear of social rejection, a person facing mild impairment doesn't seek timely medical intervention thereby leading to a delay in its detection. With a system in place, these people can take the assessment as soon as they experience the symptoms. In case of reversible dementia, early detection may assist in reversing the impairment helping the person to lead a normal life, whereas in case of irreversible Dementia, an early detection would help slowdown the progression rate.

The life expectancy of Alzheimer Disease is said to be less than 7 years [3] once detected. However with its early detection, the life expectancy for these patients can further be increased. Early detection will aid caregiver and family members to plan their timely medication and safety measures.

\subsection{Methods of Detection}

The symptoms of Cognitive impairment are subtle and varies from person to person. These symptoms can begin with physiological changes in the brain causing subsequent changes in their psychological, cognitive and behavioural skills/aspects. The behavioural and cognitive symptoms may become visible a few years before the clinical diagnosis. The progressive physiological changes in the brain can be evidenced through clinical tests and neuroimaging scans such as Cerebrospinal fluid (CSF), blood tests, electroencephalogram (EEG), magnetic resonance imaging (MRI), positron emission tomography (PET) and computerized tomography (CT) scan. Researchers have shown that these changes may aid in early detection of impairment. The decline in memory, language, thinking and judgment can be measured through a person's speech pattern, eye movements and responses to globally available neuropsychological tests. 


\section{LITERATURE REVIEW}

\subsection{Neuropsychological Assessment}

The current diagnostics use neuropsychological assessment tests based on questionnaire. These tests help determine which functions of the brain have been disrupted to what extent and what these changes mean in the person's day-to-day life. These are carried out generally after the brain injury and for the people with cognitive complaints which approximately take 10-20 minutes to administer. Author in the paper [4] reviewed list of screening tests viz. Informant based and performance based Cognitive Screening Instruments (CSI) [4, $5,9]$. In informant-based CSI, Patient's close relative is asked questions covering various cognitive domains, patient's behaviour and day to day functionality, whereas in case of performance based CSIs, patient's self-assessment report is generated.

\subsubsection{Mini Mental State Examination (MMSE)}

The cognitive tests are developed and validated to provide global assessment of cognitive states and one out of those cognitive tests commonly used is MMSE. This test is easier to score and it takes about 10 minutes to administer, however it may not capture early stages of AD. MMSE is found to be the most extensively studied psychometric screening assessment of cognitive functioning [5-10]. It is a global cognitive functioning test covering major cognitive domains such as Orientation, attention, registration, language and praxis [8]. The MMSE is mapped to different brain areas which helps to screen and classify MCI into different types of Dementia such as Fronto-temporal dementia, vascular dementia or semantic dementia etc [11]. Montreal Cognitive Assessment (MoCA) and MMSE may be of particular value for MCI diagnosis and are the most widely usedtests [12]. Table 1 shows Comparison of some of Performance based CSIs such as MoCA [12], Quick Mild Cognitive Impairment [13], and Abbreviated Mental Test Score [14] with MMSE based on various cognitive domains. In the article published by William Rodman Shanklermalet.al. [6], experimentation is done to identify the decision rules using Machine learning methods to distinguish people with early stages of dementia and normal aging. Decision tree, naive bayes and regression methods were used on 198 normal and 244 cognitively impaired persons. MMSE was also used to evaluate cognitive impairment in highly educated people [7]. In this case, an archival data were reviewed on 4248 participants enrolled in the Mayo Clinic. An overall correct classification rate of $90 \%$ was achieved in detecting dementia with the standard MMSE cut score of 27 ( 26 or below). One of the limitation of MMSE is that there is a lack of emphasis on memory ( 3 out of 30 ) and more weightage given to the orientation (10 out of 30 ) and Language ( 8 out of 30 ). Though MMSE is commonly used screening test, its result is influenced by age and education. In paper[9], MoCA seems to be better than MMSE in detecting MCI among people aged over 60 . MOCA addresses additional frontal-executive function but it is more complex to administer and score [4].

\subsubsection{Clock Drawing Test (CDT)}

MMSE requires the patient to have some basic education. On the other hand, Clock Drawing Test (CDT) is specifically designed to profile people with low literacy level. The methods of CDT are viz. free drawn, pre-drawn and set-hands method which are used to assess Visuo-spatial and executive control[16].The difference between the drawing and copying a clock can also be a measure of executive control function[18].
Table 1. Comparison of MMSE with other Cognitive Assessment Tests

\begin{tabular}{|c|c|c|c|c|}
\hline \multirow[t]{2}{*}{$\begin{array}{l}\text { Cognitive } \\
\text { Domains }\end{array}$} & \multicolumn{4}{|c|}{$\begin{array}{l}\text { Assessment Test and Weightage } \\
\text { Assigned for Cognitive Domain }\end{array}$} \\
\hline & MMSE & AMTS & $\begin{array}{c}\text { QMC } \\
\text { I }\end{array}$ & $\begin{array}{l}\text { Mo } \\
\text { CA }\end{array}$ \\
\hline Orientation & 10 & 3 & 10 & 6 \\
\hline Working Memory & 3 & 5 & 5 & 3 \\
\hline Semantic Memory & - & - & 10 & - \\
\hline $\begin{array}{c}\text { Episodic Memory } \\
\text { (Immediate, } \\
\text { Delayed Recall) }\end{array}$ & 3 & 1 & $50 *$ & 5 \\
\hline $\begin{array}{c}\text { Attention / } \\
\text { Concentration }\end{array}$ & 5 & 1 & - & 6 \\
\hline $\begin{array}{c}\text { Language } \\
\text { (Reading,Writing,V } \\
\text { erbal Fluency in } \\
\text { naming) }\end{array}$ & 8 & - & 10 & 3 \\
\hline Abstraction & - & - & - & 2 \\
\hline $\begin{array}{c}\text { Visual } \\
\text { Construction / } \\
\text { Executive function }\end{array}$ & 1 & & 15 & 5 \\
\hline Total Score & 30 & 10 & 100 & 30 \\
\hline
\end{tabular}

*Score assigned for Immediate Recall-30 and Delayed Recall $-20$

Paper [21] discusses various scoring methods of CDT and their comparative study with respect to performance and correlation with other screening tests. Here MMSE performance was found to be good when used along with CDT. While CDT was found to be useful for screening of moderate to severe dementia, it may not be so sensitive for early and mild cases. CDT scores were found to be strongly correlated with the level of executive function deficits. CDT and MMSE together detected $95 \%$ cases as abnormal as compared to $47 \%$ detected by MMSE alone [19]. This experiment was carried out for people aged over 60 with MCI and Dementia. Author of Paper [22] found that free-drawn CDT version is more cognitively demanding and sensitive for detecting mild/early cognitive impairment as against an incomplete-copy version where subjects were given a clock face with numbers and asked to copy the clock and set the hands for a specific time. Authors in the paper [15] discussed various scoring systems and mentioned 4 classes of interpreting the clock drawing viz. Bizarre clocks, major and minor spacing abnormality and normal clocks. CDT in isolation may not be an appropriate screening test to detect MCI. In another research [17], MMSE, CDT and Verbal fluency test (VFT) of 247 older persons with nearly equal number of $\mathrm{MCI}, \mathrm{AD}$ and healthy controls were carried and found that all the tests either separately or in combination of two tests worked well for identification of AD. The same even gave good specificity for distinguishing MCI from normal controls but with the low sensitivity. 


\subsection{Speech}

MCI causes slight changes in speech and language and is known to influence linguistic ability which may aggravate in later stages of disease. In the early stages, a person may find difficulty in finding or retrieving a right word in the spontaneous speech, subsequently in its later stage, followed by limited and restricted use of words. Eventually, it ends up with longer pauses, lower speech rate causing changes in emotional response too. Progression of this deterioration can be reduced if these changes are caught at its mild stages. To support this, researchers have explored temporal, acoustic and linguistic features extracted from speech to detect cognitive impairment. The goal of following literature study is to find correlation of acoustic and language features in classifying MCI, Dementia or AD from healthy controls. These features are extracted from spontaneous speech or narrative task such as picture or event description, word list, animal vegetable naming, paraphrasing etc.

The speech features are extracted using manual and automatic transcription using Automatic Speech Recognizer (ASR) trained for a particular language. First attempt [25] is proposed to extract speech and language features from spontaneous speech, which are verified using CDR (Clinical Dementia Rating) scale to evaluate accuracy for MCI diagnosis. They measured the complexity and information content of narrative story re-telling from 37 participants with MCI and 37 healthy controls. SVM classifier is used to classify the groups with an AUC of 0.73 using only speech features alone and 0.86 by combining this information with clinical test scores. A complex network graph [26] is built using distinct words as nodes which are connected by edges to their adjacent words. This method performed well for short transcripts. Acoustic features can even help distinguish between participants with MCI and elderly controls with a best accuracy of $80.4 \%$ [27]. In this case, the SVM performed better on manually extracted features than automatically extracted features using Automatic Speech Recognizer (ASR). Temporal acoustic features as given in Table 2 extracted from spontaneous speech could help distinguish MCI and Healthy control using ASR trained on Hungarian Language [28]. The vocabulary size in speech can be an early indicator of cognitive impairment [29]. The gap in content between the written and spoken narratives can also be one of the markers to distinguish between healthy and $\mathrm{AD} /$ Dementia candidates. In this case, the model was built using syntactic and lexical features from verbal utterances. Statistical analysis, NLP based techniques were carried out on written and spoken narratives of 22 participants. The gap was significantly larger in the Low Hasegawa's dementia scale (HDS-R) Group [30]. SVM outperformed over all other experimented machine learning models. The extracted language features were coordinated sentences, subordinated sentences, reduced sentences, number of predicates, average number of predicates, dependency distance, Number of dependencies, average dependencies per sentence [30]. Authors in paper [31] found that the automatic speech analysis could be an additional objective assessment tool for elderly with cognitive decline with the classification accuracy of $79 \%$ and $65 \%$ between Healthy Controls and MCI. In this paper, a Computer based analysis system is developed for detection of dementia using various acoustic and lexical features from spontaneous speech. [33]. It proved that though language impairment is a secondary cognitive marker, it provides a great insight for early Dementia detection with classification accuracy of $81 \%$ in distinguishing individuals with $\mathrm{AD}$ from those without $\mathrm{AD}$ based on short samples of their language on a picture description task. Author [23] proved that the number of filled Pauses also display a statistically significant difference between the speech of the two groups of speakers as MCI and healthy control. This can be used for early detection of disease. Table 2 shows various temporal, acoustic and linguistic features used in the research. To sum up, the speech and language features along with cognitive assessment tests is found to improve detection of cognitive impairment.

\subsection{Facial Expression}

There are some other studies carried out primarily in detection of impairment in later stages as $\mathrm{AD}$ and Dementia. Facial movements of demented patients in comfort and distress stimuli are studied to find any distinction [43]. Many people can smile out of natural frustration and this laughter and smile is detected using SVM classifier in the ROMEO2 corpus. It is a multimodal corpus having audio and video of social interaction of elderly people with Nao Robot [43]. Social gaze can also contribute as a marker of disease progression [44]. In this paper, Gaze behavior is assessed by coders. Trained coders viewed the recordings and a score of 1 was given if the participant look his/her partner's eyes. The relationship between the ability to identify the emotions and interpersonal behavior is also explored in dementia of the Alzheimer type [45]. Impairment in identifying the negative facial emotions are also experimented.

Table 2. Metrics for Speech and Eye dynamics

\begin{tabular}{|c|c|c|}
\hline Taks & $\begin{array}{l}\text { Speech } \\
\text { paramet } \\
\text { ers }\end{array}$ & $\begin{array}{c}\text { Speech } \\
\text { metrics/measures }\end{array}$ \\
\hline \multirow{3}{*}{$\begin{array}{c}\text { Speech } \\
\text { Spontaneous } \\
\text { Speech } \\
\text { Story re-telling } \\
\text { Picture / Event } \\
\text { Description } \\
\text { Animal/ } \\
\text { vegetable naming } \\
\text { Word list recall }\end{array}$} & & \\
\hline & $\begin{array}{l}\text { Temporal } \\
\text { and } \\
\text { acoustic } \\
\text { features } \\
\text { from } \\
\text { speech }\end{array}$ & $\begin{array}{l}\text { Articulation Rate, Length of } \\
\text { utterance, Silent and filled } \\
\text { Pauses, Speech rate, Stressed } \\
\text { word duration, Pauses } \\
\text { duration, no. of pauses, } \\
\text { Hesitations rate, Average } \\
\text { Syllable Duration, Speech } \\
\text { tempo, Number of phones }\end{array}$ \\
\hline & $\begin{array}{l}\text { Linguistic } \\
\text { Features }\end{array}$ & $\begin{array}{c}\text { Frequency of noun, verbs, } \\
\text { function words, words about } \\
\text { emotions, Length of } \\
\text { utterance, Wordcount, } \\
\text { unique words } \\
\text { Number of unique bigrams } \\
\text { Dependency distance, Type } \\
\text { Token Ratio, Verbal fluency }\end{array}$ \\
\hline $\begin{array}{l}\text { Eye dynamics } \\
\text { Observing a } \\
\text { visual stimuli } \\
\text { such as an image, } \\
\text { video or follow a } \\
\text { moving dot } \\
\text { Reading a } \\
\text { paragraph }\end{array}$ & $\begin{array}{c}\text { Saccadic } \\
\text { Movement } \\
\text { Fixation } \\
\text { Gaze }\end{array}$ & $\begin{array}{c}\text { Saccades Orientation, } \\
\text { Outgoing Saccade, Single } \\
\text { fixation, First pass fixation, } \\
\text { Second pass fixation, } \\
\text { Regression, Re-fixation, } \\
\text { Total duration, Total no. of } \\
\text { fixations, Total fixation time }\end{array}$ \\
\hline
\end{tabular}

The experiment is carried out on patients with Fronto temporal dementia (FTD) and AD patients. It is found that $\mathrm{AD}$ patients performed better than FTD patients in emotion 
discrimination [46]. Paper [47] built an automatic facial expression recognition system comparing similarity values of facial using the Gabor coding. Authors experimented this with six basic facial expressions (happiness, sadness, surprise, anger, disgust, fear) and a neutral face for a total of 219 images.

Depression is common in elderly people and person suffering from cognitive impairment may also have depression [34]. The automated facial image analysis and audio signal processing have been used to assess depression [48]. Author made an attempt to address change in symptom severity in a clinical sample using machine learning. The behavioural observations may indicate psychological disorders.

\subsection{Eye movements}

Research showed that episodic and semantic memory changes can be an early indicator of AD. In addition to this, some research has shown that visual attention and executive deficit can help detect the early stages of the disease [50]. Impaired visual attention can cause mistakes in perceptions in deciding depth, motion, and color of an object. Degeneration in parietal lobe can cause defects in fixation. Many researchers compared eye movements and changes in the saccade of healthy control with those of $\mathrm{AD}$ patients and were able to establish correlation of eye behavior to cognitive impairment. Eye tracking approach is used to assess the visual changes and this technique is less invasive as compared to neuroimagaing. It does not require additional patient's responses such as button presses, to make inference about physical changes. The eye trackers those are used for data collection are screen based or head mounted. In the same research, the patients were asked to fix their gaze to a point or asked to observe a visual stimulus such as an image, video or follow a moving dot. In some cases, the eye movement analysis is studied using paragraph reading.

In researches carried out so far, Saccadic Eye movements are proven to be an early indicator. Saccades are rapid eye movements that allow to quickly scan a visual scene. Saccadic movements can be of following types as when the eye movements are towards a target then it is called as prosaccades and when it is in the opposite direction, called as an antisaccades [51]. In smooth pursuit, participants continuously follow moving target object. This literature survey is to understand the eye behavior correlating MCI. It is found that participants with $\mathrm{AD}$ during reading tend to skip words and have increased number of fixations and regressions as compare to healthy controls [39]. There is a correlation of eye movement to memory, attention and executive impairment and their role in progression from MCI to $\mathrm{AD}[40]$. In the paper [41] a classification accuracy of $88.3 \%$ is reported in distinguishing between participants with $\mathrm{AD}$ and healthy controls through eye tracking measures during reading.

\section{DISCUSSION}

The neupsychological test such as MMSE can be used as a baseline. It can be drilled down further to the cognitive function with poor score, making it an adaptive test. It has been seen that the work done so far is limited to few number of participants. So far Speech systems researched are for Japanese and Hungarian language. There is a very less work been done in facial expression and in the case of image features only one feature as smiling ratio is considered for distinguishing between two groups. There are other untouched and unexplored areas like hearing, mood, emotion recognition which can be further analyzed for their influence on assessment accuracy and these timely changes would help get to the progression in the cognitive deterioration.

\section{CONCLUSION}

As the symptoms of cognitive impairment are very subtle in nature, a system integrating speech, eye movements and facial expressions is preferred. This system would be a combination of indicators reviewed in this paper assuming that the smallest change in these can contribute to its detection. Further, the current systems are invasive, expensive and have limited accessibility to frontline screening tool for Alzheimer disease. This system will be a preclinical non-invasive tool to assess the changes in cognition through various computer-based stimuli.

Further in future with the continuous use of this multimodal system, we can track the symptoms of progression into various types of dementia such as fronto-temporal, vascular, Lewy body etc. This system would record the symptoms which patients themselves and the people nearby miss.

\section{REFERENCES}

[1] Global Health and Aging - World Health Organization http://www.who.int/ageing/publications/global_health.pd $\mathrm{f}$

[2] World Alzheimer Report 2019: Attitudes to dementia. London: Alzheimer's Disease International https://www.alz.co.uk/research/WorldAlzheimerReport2 019.pdfhttps://www.alz.co.uk/research/WorldAlzheimer Report2015.pdf

[3] Dauwels J, Kannan S. "Diagnosis of Alzheimer's disease using electric signals of the brain. A grand challenge", AsiaPacific Biotech News 2012;16(10n11):22-38, http://dx.doi.org/10.1142/S0219030312000651http://ww w.asiabiotech.com/publication/apbn/16/english/preserved docs/1610n11/1610n11.pdf

[4] Breda Cullen, Brian O'Neill, Jonathan J Evans, Robert F Coen, Brian A Lawlor " A review of screening tests for cognitive impairment," J Neurol Neurosurg Psychiatry 2007;78:790-799. doi: 10.1136/jnnp.2006.095414.

[5] James E. Galvin, "Using Informant and Performance Screening Methods to Detect Mild Cognitive Impairment and Dementia", Springer Science and Business Media, LLC, part of Springer Nature 2018, 26 January 2018.

[6] William Rodman Shankle1, Subramani Mani, Michael J. Pazzani and Padhraic Smyth2,'Detecting Very Early Stages of Dementia from Normal Aging with Machine Learning Methods".

[7] Sid E. O'Bryant, Joy D. Humphreys,,"Detecting Dementia With the Mini-Mental State Examination in Highly Educated Individuals", ARCH NEUROL/VOL 65 (NO. 7), JULY 2008.

[8] M. F. Folstein, S. E. Folstein, and P. R. McHugh, "Minimental state'. A practical method for grading the cognitive state of patients for the clinician," J. Psychiatric Res., vol. 12, no. 3, pp. 189198, 1975.

[9] Natalia Ciesielska, Remigiusz Sokołowski, Ewelina Mazur, Marta Podhorecka, Anna Polak-Szabela, Kornelia Kędziora-Kornatowska,"Is the Montreal Cognitive Assessment (MoCA) test better suited than the Mini-Mental State Examination (MMSE) in mild cognitive impairment (MCI) detection among people aged over 60? Meta-analysis",J. Psychiatr. Pol. 2016; 
50(5):1039-1052,DOI:

https://doi.org/10.12740/PP/45368

[10] Andrew J. Larner, "Cognitive Screening Instruments for the diagnosis of mild cognitive Impairment", Progress in Neurology and Psychiatry March/April 2016.

[11] Nina Kachiyanys, Kye kim, "Mini mental status examination mapping to corresponding brain areas in dementia " , Applied Technologies and Innovation Journal, Vol. 7, Issue 2, pp. 55 to58. http://dx.doi.org/10.15208/ati.2012.7

[12] Nasreddine ZS, Phillips NA, Bédirian V, et al.The Montreal Cognitive Assessment, MoCA: abrief screening tool for mild cognitive impairment. J Am Geriatr Soc 2005; 53:695-9.

[13] Occas Pap R Coll Gen Pract," Abbreviated Mental Test Score (AMTS)", 1993 Apr; (59): 28.

[14] O'Caoimh, R. 2015. The Quick Mild Cognitive Impairment (Qmci) screen: developing a new screening test for mild cognitive impairment and dementia. PhD Thesis, University College Cork.

[15] Delnaz Palseia, G. Prasad Rao, Sarvada C. Tiwari1, Pragya Lodha2, Avinash De Sousa, "The Clock Drawing Test versus Mini mental Status Examination as a Screening Tool for Dementia: A Clinical Comparison ",Indian Journal of psychological Medicine Psychiatric Society - South Zonal Branch, DOI:10.4103/IJPSYM.IJPSYM_244_17,2018.

[16] Berit A, OVE DEHLJN, "REVIEW The clock drawing Test. Age and Ageing 1998; 27:399-403.

[17] Rodolfo B. Ladeira, Breno S. Diniz, Paula V. Nunes, Orestes V. Forlenza, " Combining Cognitive Screening Tests for the evaluation of MCI in the elderly ", clinics2009 doi: 10.1590/S1807-59322009001000006.

[18] Royall DR, Cordes JA, Polk M. CLOX: an executive clock drawing test. J Neurol Neurosurg Psychiatry 1998; $64: 588-94$

[19] C Mittal, Col SP Gorthi, Maj Gen S Rohatgi, VSM, "Early Cognitive Impairment: Role of Clock Drawing Test”, MJAFI Elsevier 2010.

[20] Jesus Cachoa, Julian BenitoLe,onb, Ricardo Garc 1aGarc,1ad, Bernardino FernandezCalvoe,Jose Luis VicenteVillard, onf and Alex J. Mitchellg, "Does the Combination of the MMSE and Clock Drawing Test (MiniClock) Improve the Detection of Mild Alzheimer's Disease and Mild Cognitive Impairment?", Journal of Alzheimer's Disease 22 (2010) 889-896 889,DOI 10.3233/JAD2010101182.

[21] Elisabete Pinto Ruth Peters, "Literature Review of the Clock DrawingTest as a Tool for Cognitive Screening", Dement Geriatr Cogn Disord 2009;27:201-213,DOI: $10.1159 / 000203344$

[22] Bárbara Costa Beber1, Renata Kochhann1,2, Bruna Matias1, Márcia Lorena Fagundes Chaves1,3, "The Clock Drawing Test Performance differences between the free-drawn and incomplete-copy versions in patients with MCI and dementia" pubmed Dement Neuropsychol. 2016 Jul-Sep;10(3):227-231 Berit A. The clock drawing Test. Age and Ageing 1998; 27 :399-403.

[23] K. L. de Ipi na, J.-B. Alonso, C.M. Travieso, J. Sol-
Casals, H. Egiraun,M. Faundez-Zanuy, A. Ezeiza, N. Barroso, M. Ecay-Torres,P. Martinez-Lage, and U. M. de Lizardui, "On the selection of non-invasive methods based on speech analysis oriented to automatic Alzheimer disease diagnosis," Sensors, vol. 13, no. 5, pp. 6730-6745, 2013.

[24] [24] [11b] A. König et al., "Automatic speech analysis for the assessment of patients with predementia and Alzheimer's disease," Alzheimer's Dementia, Diagnosis, Assessment Disease Monitor., vol. 1, no. 1, pp. 112124, 2015.

[25] B. Roark, M. Mitchell, J.-P. Hosom, K. Hollingshead, and J. Kaye, "Spoken language derived measures for detecting mild cognitive impairment," IEEE Trans. Audio, Speech, Language Process., vol. 19, no. 7,pp. 20812-090, Sep. 2011.

[26] Leandro B. dos Santos1, Edilson A. Corr^ea Jr1, Osvaldo N. Oliveira Jr2, Diego R. Amancio1,Let'icia L. Mansur3, Sandra M. Alu'1sio, "Enriching Complex Networks withWord Embeddings for Detecting Mild Cognitive Impairment from Speech Transcripts", arXiv:1704.08088v1 [cs.CL] 26 Apr 2017

[27] L. T'oth, G. Gosztolya, V. Vincze, I. Hoffmann, G. Szatl'oczki, E. Bir'o, F. Zsura, M. P'ak'aski, and J. K'alm'an, "Automatic detection of mild cognitive impairment from spontaneous speech using ASR," in Proceedings of Interspeech, Dresden, Germany, Sep 2015, pp. 2694-2698.

[28] Nikhil Yadav,Christian Poellabauer,Louis Daudet, "Portable Neurological Disease Assessment UsingTemporal Analysis of Speech", BCB'15, September 09-12, 2015, Atlanta, GA, USA.

[29] E. Aramaki, S. Shikata, M. Miyabe, and A. Kinoshita, "Vocabulary size in speech may be an early indicator of cognitive impairment," PloS ONE, vol. 11, no. 5, p. E0155-195, 2016.

[30] S. O. Orimaye, J. S.-M. Wong, and K. J. Golden, "Learning predictive linguistic features for Alzheimer's disease and related dementias using verbal utterances", Association for Computational Linguistics , 2014.

[31] Jarrold, Bart Peintner, David Wilkins, Dimitra Vergryi and Colleen Richey, Maria Luisa Gorno-Tempini and Jennifer Ogar, "Aided Diagnosis of Dementia Type through Computer-Based Analysis of Spontaneous Speech", Workshop on Computational Linguistics and Clinical Psychology: From Linguistic Signal to Clinical Reality, pages 27-37, Baltimore, Maryland USA, June 27, 2014

[32] K. C. Fraser, J. A. Meltzer, and F. Rudzicz, "Linguistic features identify Alzheimer's disease in narrative speech," J. Alzheimer's Disease, vol. 49, no. 2, pp. 407422, 2015.

[33] G'abor Gosztolya 1,2, L'aszl'o T'oth 1, Tam'as Gr'osz 2, Veronika Vincze 1,Ildik'o Hoffmann 34, Gr'eta Szatl'oczki 5, Magdolna P'ak'aski 5, J'anos K'alm'an 5, "Detecting Mild Cognitive Impairment from Spontaneous Speech by Correlation-Based Phonetic Feature Selection", INTERSPEECH 2016 September 812, 2016, San Francisco, USA.

[34] J. F. Cohn et al., "Detecting depression from facial actions and vocal prosody", Proc. 3rd Int. Conf. 
Affective Comput. Intell. Interact. Workshops (ACII), pp. 1-7, Sep. 2009.

[35] H Tanaka, H Adachi, N Ukita, M Ikeda, H Kazui, T kudo, and S Nakamura, "Detecting Dementia Through Interactive Computer Avatars", IEEE journal of Translational Engineering in Health and Medicine, 2017.

[36] Ane Alberdi Aramendi, Asier Aztiria, Adrian Basarab, "On the early diagnosis of Alzheimer 's disease from multimodal signals: A survey", Artificial Intelligence in Medicine, vol. 71.pp. 1-29. ISSN 0933-3657,2016

[37] [37] S C Tiwari, Rakesh Kumar Tripathi Aditya Kumar, " Applicability of the Mini-mental State Examination (MMSE) and the Hindi Mental State Examination (HMSE) to the urban elderly in India: A pilot study, December 2008International Psychogeriatrics 21(1):1238 DOI: $10.1017 / \mathrm{S} 1041610208007916$

[38] Anderson TJ, MacAskill MR., "Eye movements in patients with neurodegenerative disorders. Nat Rev Neurol 2013;9(2):7485,http://dx.doi.org/10.1038/nrneurol.2012.273 http://www.ncbi.nlm.nih.gov/pubmed/23338283

[39] Gerardo Fernandez, Pablo Mandolesi, Nora P Rotstein, Oscar Colombo, Osvaldo Agamennoni, and Luis E Politi., "Eye movement alterations during reading in patients with early Alzheimer disease", Investigative ophthalmology \& Visual Science,2013 54(13):83458352.

[40] Marta LG Pereira, Marina von Zuben A Camargo, Ivan Aprahamian, and Orestes V Forlenza, "Eye movement analysis and cognitive processing: detecting indicators of conversion to Alzheimer's disease",Neuropsychiatric Disease and Treatment, 2014,10:1273-1285.

[41] Juan Biondi, Gerardo Fernandez, Silvia Castro, and Osvaldo Agamenonni. 2017. Eye-movement behavior identification for $\mathrm{AD}$ diagnosis. arXiv preprint arXiv: 1702.0083

[42] Kathleen C. Fraser1 , Kristina Lundholm Fors1 , Dimitrios Kokkinakis1, Arto Nordlund2 "An analysis of eye-movements during reading for the detection of mild cognitive impairment", Proceedings of the 2017
Conference on Empirical Methods in Natural Language Processing, pages 1016-1026 Copenhagen, Denmark, September 7-11, 2017. C 2017 Association for Computational Linguistics.

[43] M. Hoque, R. W. Picard, "Acted vs. Natural frustration and delight: Many people smile in natural frustration", Proc. IEEE Int. Conf. Autom. Face Gesture Recognit. Workshops (FG), pp. 354-359, Mar. 2011.

[44] K. Asplund, A. Norberg, R. Adolfsson, H. M. Waxman, "Facial expressions in severely demented patients-A stimulus-response study of four patients with dementia of the Alzheimer type", Int. J. Geriatric Psychiatry, vol. 6, no. 8, pp. 599-606, 1991.

[45] V. E. Sturm et al., "Mutual gaze in Alzheimer's disease frontotemporal and semantic dementia couples", Social Cognit. Affective Neurosci., vol. 6, no. 3, pp. 359-367, 2010.

[46] A. Shimokawa et al., "Influence of deteriorating ability of emotional comprehension on interpersonal behavior in alzheimer-type dementia", Brain cognition, vol. 47, no. 3, pp. 423-433, 2001.

[47] D. Fernandez-Duque, S. E. Black, "Impaired recognition of negative facial emotions in patients with frontotemporal dementia", Neuropsychologia, vol. 43, no. 11, pp. 1673-1687, 2005.

[48] M. Lyons, S. Akamatsu, M. Kamachi, J. Gyoba, "Coding facial expressions with Gabor wavelets", Proc. 3rd IEEE Int. Conf. Autom. Face Gesture Recognit., pp. 200-205, Apr. 1998.

[49] J. F. Cohn et al., "Detecting depression from facial actions and vocal prosody", Proc. 3rd Int. Conf. Affective Comput. Intell. Interact. Workshops (ACII), pp. 1-7, Sep. 2009.

[50] Tim J. Anderson and Michael R. MacAskill, "Eye movements in patients with neurodegenerative disorders", Macmillan Publishers Limited, VOL 9. FEBRUARY 2013

[51] R. J. Molitor, P. C. Ko, and B. A. Ally, "Eye movements in Alzheimer's disease," Journal of Alzheimers Disease, vol. 44 , no. 1, pp. 1-12, 2015. 